\title{
New approximate formula for the arrhenius temperature integral by using incomplete gamma functions
}

\author{
Erhan Eser a , Hüseyin Koç b, ${ }^{*}$ \\ ${ }^{a}$ Hacı Bayram Veli University, Department of Physics, 06900, Ankara Turkey \\ b Muş Alparslan University, Department of Electrical and Electronics Engineering, 49250, Muş Turkey
}

AR T I CLE INFO

Article history:

Received 22 February 2019

Received in revised form 24 June 2019

Accepted 24 June 2019

Keywords:

Arrhenius integral

Kinetics reaction,

Non-isothermal kinetic

Incomplete gamma function

\begin{abstract}
A B S T R AC T
A new analytical formula for the Arrhenius integral has been proposed by using incomplete gamma functions, which is simple, accurate and reliable. The proposed formula has compared with several published the Arrhenius integral approaches, and is in agreement with the other approaches. Compared with the other approximate formulas proposed in the literature, our proposed method gives more accurate values in the precision of the activation energy as a function of $x$ and temperature.
\end{abstract}

\section{Introduction}

Many technologically important chemical reactions and processes occur under non-isothermal conditions. Also, it is reaction over time during transient heating. Integral methods used for non-isothermal analysis use cumulative values determined in temperature scanning experiment to obtain the kinetic parameters of a reacting system and find a suitable rate law [1-7]. Although integral methods purposed in the literature [3-17] are useful, problems of the integral methods are that they have low sensitivity and a little cumbersome mathematic for the evaluation of the Arrhenius integral, which does not have an exact analytical solution, found in the analyses. In this study, new analytical formulas are obtained using incomplete gamma functions. It is shown that the proposed analytical method is a simple, reliable and ideal solution for the evaluation of the Arrhenius integral.

where $A$ is the pre-exponential factor of Arrhenius, $E$ is the activation energy and $R$ is the gas constant. The integral of Arrhenius equation usually is necessary to make kinetic analysis of experimental data obtained under isothermal conditions. Thus, if the temperature is a known function of the time, the conversional fraction also depends on the temperature. In this context, Eq.(1) cannot be directly

\section{Theoretical Method}

The temperature dependence of the reaction rate for the thermal degradation can be described by the following equation [18]:

$\frac{d \alpha}{d t}=\kappa(T) f(\alpha)$

where $d \alpha / d t$ is the rate of the reaction in a certain instant $t$ and $f(\alpha)$ is a function depending on the reaction mechanism and $\kappa(T)$ is a temperature-dependent rate constant, given by the equation:

$\kappa(T)=A \exp \left(-\frac{E}{R T}\right)$

integrated with increasing temperature unless it is known the temperature-dependence function of temperature. Supposing the temperature variation is conducted at a constant heating rate, $\beta=d T / d t$, Eq. (1) can be written as:

$\frac{d \alpha}{d T}=\frac{A}{\beta} \exp \left(-\frac{E}{R T}\right) f(\alpha)$ 
With variable separation and integration, Eq. (3) can be written as

$$
g(\alpha)=\int_{0}^{\alpha} \frac{d \alpha}{f(\alpha)}=\frac{A}{\beta} \int_{0}^{T} \exp \left(-\frac{E}{R T}\right)
$$

or

$g(\alpha)=\left(\frac{A}{\beta}\right) \frac{E}{R} I_{\eta}(x, \mu)$

Where $x=E / R T$, the function $I_{\eta}(x, \mu)$ (for $\mu=-2$ and $\eta=1$ ) is defined by the following integral:

$I_{\eta}(x, \mu)=\int_{x}^{\infty} x^{\mu} e^{-\eta x} d x$

In above, $g(\alpha)$ is the integral conversion function and allows fort direct analysis of experimental data which is obtained from the measurement of the expansion of an integral magnitude such as the mass loss recorded at thermal equilibrium as a function of the temperature under a linear heating program.

The integral of the Arrhenius equation $\left(I_{\eta}(x, \mu)\right)$ does not have an exact analytical solution, and several approximated analytical solutions have been reported in the literature to determine the activation energy of solid-state reactions from the experimental data $[6,7,11,13-15]$.

It is seen that the Arrhenius integral (Eq.(6)) can be expressed by incomplete gamma function. In literature, there are a number useful methods for evaluating the incomplete gamma function. Thus, the problem in determination of the Arrhenius integral is reduced to the calculation of basic integrals such as the incomplete gamma function. Thus, from above equations, the following expressions for the Arrhenius integral can be obtained:

$$
I(\mu, x)=\left\{\begin{array}{lr}
(-1)^{\mu} \frac{1}{(|\mu|-1) !} E_{i}(x)+\frac{e^{-x}}{x^{|\mu|}} \sum_{k=1}^{|\mu|-1} \frac{(-1)^{k-1} x^{-k}}{k ! F_{k}(|\mu|-1)}, & \text { for negaitive integer } \mu \\
\Gamma(\mu+1, x), & \text { for noninteger and negaitive } \mu \\
\frac{\Gamma(\mu+2, x)-e^{-x} x^{\mu+1}}{\mu+1}, & \text { for negaitive } \mu \text { and } \mu \neq 1
\end{array}\right.
$$

Where $x \neq 0$ and $F_{m}(n)$ is the binomial coefficient and $\Gamma(\alpha, x)$ is the well-known incomplete gamma functions defined by [19],

$\Gamma(\alpha, x)=\int_{x}^{\infty} t^{\alpha-1} e^{-t} d t$

In Eq.(6), $I(\mu, x)$ for $\mu>0$ ve $\mu=0$ is as follows:

$I(\mu, x)=e^{-x} \sum_{k=0}^{\mu} \frac{\mu ! x^{k}}{k !} \quad$ for positive $\mu$ and $\mu=0$

Also, the Arrhenius integral obtained from the Mathematica 8.0 international mathematical software is given below:

$$
\begin{aligned}
& I(-2, x)=\int_{x}^{\infty} t^{-2} e^{-x} d x= E i(-x)+\frac{e^{-x}}{x} \\
& \varepsilon \%=\left\{\begin{array}{l}
\left(-\frac{\partial \operatorname{In}(I(x))}{\partial x}-\frac{2}{x}-1\right) 100 \\
\left(-\frac{0.4343}{0.4567}\left(\frac{\partial \operatorname{In}(I(x))}{\partial x}\right)-1\right) 100 \\
\left(-\frac{\partial \operatorname{In}(I(x))}{\partial x}-1\right) 100 \\
\left(-\frac{\partial \operatorname{In}(I(x))}{\partial x}-\frac{1}{x}-1\right) 100
\end{array}\right.
\end{aligned}
$$

Coats and Redfern method

Doyle method

Horowitz and Metzger method

Van Krevelen method
The function of integral $\mu$, which is defined in Eqs.(7)-(9), were evaluated in the range of $x=0.1-15$. The obtained results with the literature data have been given in Table 1. Besides, the calculations of the Eqs.(7)-(9) and (12) for the range of $x=0-$ 12000 are shown in Table 2.

\section{Errors in the Activation Energy}

In the literature, some relative error approximations to the Arrhenius integral as a function of the parameter of $x$ (E/RT) have been proposed $[6,7,11,13]$. Considering these approximations to the Arrhenius integral, the relative errors were obtained. In this context, the expressions for some proposed approximations to the relative error is the following: 
Table 1. Values of the $\mu$.order exponential integral functions, defined in equations 7,8 and 9

- Eq(7) ve (8), "Eq(7)-(8) ve (9)

\begin{tabular}{|c|c|c|c|c|c|c|c|c|c|}
\hline \multirow{2}{*}{$x$} & \multicolumn{2}{|c|}{$\mu=-1$} & \multicolumn{2}{|c|}{$\mu=-2$} & \multicolumn{3}{|c|}{$\mu=-1$} & \multicolumn{2}{|c|}{$\mu=-2$} \\
\hline & Ref.[20] & This Study" & Ref.[20] & This Study $^{* *}$ & $x$ & Ref.[20] & This Study* & $\begin{array}{l}\text { Ref.[20] } \\
\end{array}$ & This Study \\
\hline 0.1 & 1.823 & 1.8229 & 7.23 & 7.2255 & 7.2 & $9.22 \mathrm{E}-05$ & $9.2188 \mathrm{E}-04$ & $1.150 \mathrm{E}-05$ & 1.1504 E-05 \\
\hline 0.2 & 1.223 & 1.2226 & 2.87 & 2.8710 & 7.4 & $7.36 \mathrm{E}-05$ & $7.3639 \mathrm{E}-05$ & 8.96 E-06 & 8.9620 E-06 \\
\hline 0.3 & $9.06 \mathrm{E}-01$ & $9.0567 \mathrm{E}-01$ & 1.563 & 1.5637 & 7.6 & $5.89 \mathrm{E}-05$ & $5.8859 \mathrm{E}-05$ & $6.99 \mathrm{E}-06$ & $6.9901 \mathrm{E}-06$ \\
\hline 0.4 & $7.02 \mathrm{E}-01$ & $7.0238 \mathrm{E}-01$ & $9.74 \mathrm{E}-01$ & $9.7342 \mathrm{E}-01$ & 7.8 & $4.71 \mathrm{E}-05$ & $4.7072 \mathrm{E}-05$ & $5.46 \mathrm{E}-06$ & $5.4585 \mathrm{E}-06$ \\
\hline 0.5 & $5.60 \mathrm{E}-01$ & $5.5977 \mathrm{E}-01$ & $6.52 \mathrm{E}-01$ & $6.5329 \mathrm{E}-01$ & 8 & 3.77 E-05 & $3.7666 \mathrm{E}-05$ & $4.27 \mathrm{E}-06$ & $4.2672 \mathrm{E}-06$ \\
\hline 0.6 & $4.54 \mathrm{E}-01$ & $4.5438 \mathrm{E}-01$ & $4.61 \mathrm{E}-01$ & $4.6031 \mathrm{E}-01$ & 8.2 & $3.02 \mathrm{E}-05$ & $3.0155 \mathrm{E}-05$ & 3.34 E-06 & $3.3395 \mathrm{E}-06$ \\
\hline 0.7 & 3.74 E-01 & $3.7376 \mathrm{E}-01$ & 3.35 E-01 & 3.3564 E-01 & 8.4 & $2.42 \mathrm{E}-05$ & $2.4154 \mathrm{E}-05$ & $2.62 \mathrm{E}-06$ & $2.6161 \mathrm{E}-06$ \\
\hline 08 & 3.11 E-01 & $3.1059 \mathrm{E}-01$ & 2.51 E-01 & 2.5107 E-01 & 8.6 & $1.936 \mathrm{E}-05$ & $1.9356 \mathrm{E}-05$ & 2.05 E-06 & $2.0514 \mathrm{E}-06$ \\
\hline 09 & $2.60 \mathrm{E}-01$ & $2.6018 \mathrm{E}-01$ & $1.916 \mathrm{E}-01$ & $1.9156 \mathrm{E}-01$ & 8.8 & $1.552 \mathrm{E}-05$ & $1.5519 \mathrm{E}-05$ & 1.610 E-06 & $1.6101 \mathrm{E}-06$ \\
\hline 1 & $2.19 \mathrm{E}-01$ & $2.1938 \mathrm{E}-01$ & $1.485 \mathrm{E}-01$ & $1.4849 \mathrm{E}-01$ & 9 & $1.245 \mathrm{E}-05$ & $1.2447 \mathrm{E}-05$ & $1.265 \mathrm{E}-06$ & $1.2648 \mathrm{E}-06$ \\
\hline 1.2 & $1.584 \mathrm{E}-01$ & $1.5841 \mathrm{E}-01$ & $9.26 \mathrm{E}-02$ & $9.2588 \mathrm{E}-02$ & 9.2 & 9.99 E-06 & $9.9881 \mathrm{E}-06$ & 9.95 E-07 & 9.9447 E-07 \\
\hline 1.4 & $1.162 \mathrm{E}-01$ & $1.1622 \mathrm{E}-01$ & $5.99 \mathrm{E}-02$ & $5.9921 \mathrm{E}-02$ & 9.4 & 8.02 E-06 & $8.0179 \mathrm{E}-06$ & 7.82 E-07 & $7.8254 \mathrm{E}-07$ \\
\hline 1.6 & 8.63 E-02 & $8.6308 \mathrm{E}-02$ & 3.99 E-02 & 3.9877 E-02 & 9.6 & 6.44 E-06 & $6.4388 \mathrm{E}-06$ & $6.16 \mathrm{E}-07$ & $6.1625 \mathrm{E}-07$ \\
\hline 1.8 & 6.47 E-02 & $6.4713 \mathrm{E}-02$ & 2.71 E-02 & $2.7119 \mathrm{E}-02$ & 9.8 & 5.17 E-06 & $5.1728 \mathrm{E}-06$ & 4.85 E-07 & $4.8566 \mathrm{E}-07$ \\
\hline 2 & $4.89 \mathrm{E}-02$ & $4.8901 \mathrm{E}-02$ & 1.877 E-02 & $1.8767 \mathrm{E}-02$ & 10 & $4.16 \mathrm{E}-06$ & $4.1569 \mathrm{E}-06$ & 3.83 E-07 & $3.8302 \mathrm{E}-07$ \\
\hline 2.2 & 3.72 E-02 & $3.7191 \mathrm{E}-02$ & $1.317 \mathrm{E}-02$ & 1.3174 E-02 & 10.2 & 3.34 E-06 & $3.1419 \mathrm{E}-06$ & 3.02 E-07 & $3.0229 \mathrm{E}-07$ \\
\hline 2.4 & $2.84 \mathrm{E}-02$ & $2.8440 \mathrm{E}-02$ & $9.36 \mathrm{E}-03$ & $9.3589 \mathrm{E}-03$ & 10.4 & 2.69 E-06 & $2.6875 \mathrm{E}-06$ & 2.39 E-07 & $2.3873 \mathrm{E}-07$ \\
\hline 2.6 & $2.19 \mathrm{E}-02$ & $2.1850 \mathrm{E}-02$ & 6.72 E-03 & $6.7165 \mathrm{E}-03$ & 10.6 & $2.16 \mathrm{E}-06$ & $2.1691 \mathrm{E}-06$ & $1.887 \mathrm{E}-07$ & $1.8866 \mathrm{E}-07$ \\
\hline 2.8 & $1.686 \mathrm{E}-02$ & $1.6855 \mathrm{E}-02$ & $4.86 \mathrm{E}-03$ & $4.8626 \mathrm{E}-03$ & 10.8 & 1.740 E-06 & $1.7397 \mathrm{E}-06$ & $1.492 \mathrm{E}-07$ & $1.4918 \mathrm{E}-07$ \\
\hline 3 & $1.305 \mathrm{E}-02$ & $1.3048 \mathrm{E}-02$ & $3.55 \mathrm{E}-03$ & $3.5473 \mathrm{E}-03$ & 11 & $1.400 \mathrm{E}-06$ & $1.4003 \mathrm{E}-06$ & 1.180 E-07 & $1.1804 \mathrm{E}-07$ \\
\hline 3.2 & $1.013 \mathrm{E}-02$ & $1.0133 \mathrm{E}-02$ & $2.60 \mathrm{E}-03$ & $2.6052 \mathrm{E}-03$ & 11.2 & $1.127 \mathrm{E}-06$ & $1.1275 \mathrm{E}-06$ & $9.35 \mathrm{E}-08$ & $9.3448 \mathrm{E}-08$ \\
\hline 3.4 & $7.89 \mathrm{E}-03$ & $7.8909 \mathrm{E}-03$ & $1.925 \mathrm{E}-03$ & $1.9247 \mathrm{E}-03$ & 11.4 & $9.08 \mathrm{E}-07$ & $9.0804 \mathrm{E}-07$ & 7.40 E-08 & $7.4023 \mathrm{E}-08$ \\
\hline 3.6 & 6.16 E-03 & $6.1604 \mathrm{E}-03$ & $1.430 \mathrm{E}-03$ & $1.4295 \mathrm{E}-03$ & 11.6 & $7.31 \mathrm{E}-07$ & $7.3151 \mathrm{E}-07$ & 5.87 E-08 & 5.8667 E-08 \\
\hline 3.8 & $4.82 \mathrm{E}-03$ & $4.8203 \mathrm{E}-03$ & $1.067 \mathrm{E}-03$ & $1.0668 \mathrm{E}-03$ & 11.8 & $5.89 \mathrm{E}-07$ & $5.8946 \mathrm{E}-07$ & $4.65 \mathrm{E}-08$ & $4.6522 \mathrm{E}-08$ \\
\hline 4 & $3.78 \mathrm{E}-03$ & $3.7794 \mathrm{E}-03$ & 8.00 E-04 & $7.9956 \mathrm{E}-04$ & 12 & 4.75 E-07 & $4.7511 \mathrm{E}-07$ & 3.69 E-08 & $3.6909 \mathrm{E}-08$ \\
\hline 4.2 & 2.97 E-03 & $2.9688 \mathrm{E}-03$ & 6.02 E-04 & $6.0161 \mathrm{E}-04$ & 12.2 & 3.83 E-07 & 3.8304 E-07 & 2.93 E-08 & $2.9298 \mathrm{E}-08$ \\
\hline 4.4 & 2.34 E-03 & $2.3360 \mathrm{E}-03$ & $4.54 \mathrm{E}-04$ & $4.5429 \mathrm{E}-04$ & 12.4 & $3.08 \mathrm{E}-07$ & $3.0888 \mathrm{E}-07$ & $1.848 \mathrm{E}-08$ & $2.3267 \mathrm{E}-08$ \\
\hline 4.6 & $1.841 \mathrm{E}-03$ & $1.8410 \mathrm{E}-03$ & 3.44 E-04 & 3.4418 E-04 & 12.6 & 2.49 E-07 & $2.4913 \mathrm{E}-07$ & $1.469 \mathrm{E}-08$ & $1.8486 \mathrm{E}-08$ \\
\hline 4.8 & $1.453 \mathrm{E}-03$ & $1.4529 \mathrm{E}-03$ & $2.62 \mathrm{E}-04$ & $2.6154 \mathrm{E}-04$ & 12.8 & $2.01 \mathrm{E}-07$ & $2.0099 \mathrm{E}-07$ & $1.165 \mathrm{E}-08$ & $1.4694 \mathrm{E}-08$ \\
\hline 5 & $1.148 \mathrm{E}-03$ & $1.1483 \mathrm{E}-03$ & $1.993 \mathrm{E}-04$ & $1.9929 \mathrm{E}-04$ & 13 & $1.622 \mathrm{E}-07$ & $1.6219 \mathrm{E}-07$ & $1.025 \mathrm{E}-08$ & $1.1685 \mathrm{E}-08$ \\
\hline 5.2 & $9.09 \mathrm{E}-04$ & $9.0862 \mathrm{E}-04$ & $1.523 \mathrm{E}-04$ & $1.5226 \mathrm{E}-04$ & 13.2 & $1.309 \mathrm{E}-07$ & $1.3090 \mathrm{E}-07$ & $9.30 \mathrm{E}-09$ & $9.2959 \mathrm{E}-09$ \\
\hline 5.4 & 7.20 E-04 & $7.1980 \mathrm{E}-04$ & $1.166 \mathrm{E}-04$ & $1.1659 \mathrm{E}-04$ & 13.4 & $1.057 \mathrm{E}-07$ & $1.0567 \mathrm{E}-07$ & 7.40 E-09 & 7.3985 E-09 \\
\hline 5.6 & $5.71 \mathrm{E}-04$ & $5.7084 \mathrm{E}-04$ & 8.95 E-05 & 8.9493 E-05 & 13.6 & $8.53 \mathrm{E}-08$ & $8.5322 \mathrm{E}-08$ & $5.89 \mathrm{E}-09$ & $5.8908 \mathrm{E}-09$ \\
\hline 5.8 & $4.53 \mathrm{E}-04$ & $4.5316 \mathrm{E}-04$ & $6.88 \mathrm{E}-05$ & $6.8831 \mathrm{E}-05$ & 13.8 & $6.89 \mathrm{E}-08$ & $6.8905 \mathrm{E}-08$ & $4.69 \mathrm{E}-09$ & $4.6919 \mathrm{E}-09$ \\
\hline 6 & $3.60 \mathrm{E}-04$ & $3.6008 \mathrm{E}-04$ & $5.30 \mathrm{E}-05$ & $5.3043 \mathrm{E}-05$ & 14 & $5.57 \mathrm{E}-08$ & $5.5656 \mathrm{E}-08$ & 3.74 E-09 & $3.7386 \mathrm{E}-09$ \\
\hline 6.2 & 2.86 E-04 & $2.8638 \mathrm{E}-04$ & $4.10 \mathrm{E}-05$ & $4.0951 \mathrm{E}-05$ & 14.2 & $4.50 \mathrm{E}-08$ & $4.4964 \mathrm{E}-08$ & $2.98 \mathrm{E}-09$ & 2.9800 E-09 \\
\hline 6.4 & $2.28 \mathrm{E}-04$ & 2.2795 E- 04 & 3.17 E- 05 & 3.1670 E-05 & 14.4 & $3.63 \mathrm{E}-08$ & $3.6331 \mathrm{E}-08$ & $2.38 \mathrm{E}-09$ & $2.3762 \mathrm{E}-09$ \\
\hline 6.6 & $1.816 \mathrm{E}-04$ & $1.8158 \mathrm{E}-04$ & 2.45 E-05 & $2.4533 \mathrm{E}-05$ & 14.6 & 2.94 E-08 & $2.9362 \mathrm{E}-08$ & $1.896 \mathrm{E}-09$ & $1.8954 \mathrm{E}-09$ \\
\hline 6.8 & $1.448 \mathrm{E}-04$ & 1.4476 E- 04 & $1.903 \mathrm{E}-05$ & $1.9033 \mathrm{E}-05$ & 14.8 & 2.37 E-08 & $2.3733 \mathrm{E}-08$ & $1.513 \mathrm{E}-09$ & $1.5124 \mathrm{E}-09$ \\
\hline 7 & $1.155 \mathrm{E}-04$ & $1.1548 \mathrm{E}-04$ & $1.479 \mathrm{E}-05$ & $1.4787 \mathrm{E}-05$ & 15 & $1.919 \mathrm{E}-08$ & $1.9186 \mathrm{E}-08$ & $1.207 \mathrm{E}-09$ & $1.2072 \mathrm{E}-09$ \\
\hline
\end{tabular}

Table 2. Values of Arrhenius integral calculated by different approximations

\begin{tabular}{|c|c|c|c|c|c|c|}
\hline$x$ & Eq.(7) & Eq.(8) & Eq.(9) & Eq. (11) & Ref. [3] & Ref. [4] \\
\hline 1 & $1.48495506775922 \mathrm{E}-01$ & $1.48495506775922 \mathrm{E}-01$ & $1.48495506775922 \mathrm{E}-01$ & $1.48495506775922 \mathrm{E}-01$ & 1.47240337224585 E-01 & 1.68032245935143 E-01 \\
\hline 2 & 1.87671309102452 E-02 & $1.87671309102452 \mathrm{E}-02$ & $1.87671309102452 \mathrm{E}-02$ & 1.87671309102452 E-02 & $1.87631313749237 \mathrm{E}-02$ & 1.94070574358876 E-02 \\
\hline 4 & 7.99557312334639 E-04 & 7.99557312334639 E-04 & $7.99557312334639 \mathrm{E}-04$ & 7.99557312334639 E-04 & 7.99599605275245 E-04 & 8.03428247667680 E-04 \\
\hline 5 & 1.99293808541768 E-04 & 1.99293808541768 E-04 & 1.99293808541768 E-04 & 1.99293808541768 E-04 & 1.99296763379962 E-04 & 1.99666324045517 E-04 \\
\hline 10 & 3.83024046563161 E-07 & 3.83024046563161 E-07 & 3.83024046563161 E-07 & 3.83024046563161 E-07 & 3.83023113636501 E-07 & 3.82844890862867 E-07 \\
\hline 15 & 1.20720911197638 E-09 & 1.20720911197638 E-09 & 1.20720911197638 E-09 & 1.20720911197638 E-09 & 1.20721311456225 E-09 & 1.20684895246369 E-09 \\
\hline 20 & $4.70242821542907 \mathrm{E}-12$ & $4.70242821542907 \mathrm{E}-12$ & $4.70242821542907 \mathrm{E}-12$ & $4.70242821542907 \mathrm{E}-12$ & $4.70244036514273 \mathrm{E}-12$ & 4.70184861286925 E-12 \\
\hline 25 & 2.06277790645392 E-14 & $2.06277790645392 \mathrm{E}-14$ & $2.06277790645392 \mathrm{E}-14$ & 2.06277790645392 E-14 & $2.06277902799921 \mathrm{E}-14$ & 2.06274403641353 E-14 \\
\hline 30 & 9.76556455912457 E-17 & 9.76556455912457 E-17 & $9.76556455912457 \mathrm{E}-17$ & 9.76556455912457 E-17 & 9.76555352378880 E-17 & 9.76601638134280 E-17 \\
\hline 35 & 4.87559924758312 E-19 & 4.87559924758312 E-19 & 4.87559924758312 E-19 & 4.87559924758312 E-19 & 4.87558871363914 E-19 & $4.8760048067650 \mathrm{E}-19$ \\
\hline
\end{tabular}


Bitlis Eren University Journal of Science and Technology 9(1) (2019) 27-31

\begin{tabular}{|c|c|c|c|c|c|c|}
\hline 40 & $2.53153023712403 \mathrm{E}-21$ & $2.53153023712403 \mathrm{E}-21$ & $2.53153023712403 \mathrm{E}-21$ & $2.53153023712403 \mathrm{E}-21$ & $2.53152356934944 \mathrm{E}-21$ & $2.53179585500996 \mathrm{E}-21$ \\
\hline 50 & $7.42356663773765 \mathrm{E}-26$ & $7.42356663773765 \mathrm{E}-26$ & 7.42356663773765 E-26 & $7.42356663773765 \mathrm{E}-26$ & 7.42354827362144 E-26 & $7.42449025254796 \mathrm{E}-26$ \\
\hline 60 & $2.35508947681633 \mathrm{E}-30$ & $2.35508947681633 \mathrm{E}-30$ & 2.35508947681633 E-30 & $2.35508947681633 \mathrm{E}-30$ & 2.35508585229467 E-30 & 2.35539276235031 E-30 \\
\hline 70 & $7.89076226274856 \mathrm{E}-35$ & $7.89076226274856 \mathrm{E}-35$ & $7.89076226274856 \mathrm{E}-35$ & $7.89076226274856 \mathrm{E}-35$ & $7.89075977828676 \mathrm{E}-35$ & 7.89176377843458 E-35 \\
\hline 80 & $2.75209761182961 \mathrm{E}-39$ & $2.75209761182961 \mathrm{E}-39$ & $2.75209761182961 \mathrm{E}-39$ & $2.75209761182961 \mathrm{E}-39$ & 2.75210026808223 E-39 & 2.75243475035727 E-39 \\
\hline 90 & $9.89844122331617 \mathrm{E}-44$ & $9.89844122331617 \mathrm{E}-44$ & 9.89844122331617 E-44 & $9.89844122331617 \mathrm{E}-44$ & 9.89846294745012 E-44 & $9.89960059115088 \mathrm{E}-44$ \\
\hline 100 & $3.64782143388038 \mathrm{E}-48$ & $3.64782143388038 \mathrm{E}-48$ & 3.64782143388038 E-48 & $3.64782143388038 \mathrm{E}-48$ & $3.64783357902046 \mathrm{E}-48$ & 3.64822828018871 E-48 \\
\hline 120 & $5.23815730901561 \mathrm{E}-57$ & 5.23815730901561 E-57 & 5.23815730901561 E-57 & $5.23815730901561 \mathrm{E}-57$ & 5.23818492232495 E-57 & 5.23868562268789 E-57 \\
\hline 150 & 3.14724088827775 E-70 & 3.14724088827775 E-70 & 3.14724088827775 E-70 & 3.14724088827775 E-70 & $3.14726430906919 \mathrm{E}-70$ & 3.14751559753650 E-70 \\
\hline 180 & $2.04962923472761 \mathrm{E}-83$ & $2.04962923472761 \mathrm{E}-83$ & $2.04962923472761 \mathrm{E}-83$ & $2.04962923472761 \mathrm{E}-83$ & $2.04964754842281 \mathrm{E}-83$ & $2.04978609508623 \mathrm{E}-83$ \\
\hline 200 & 3.42565273760521 E-92 & 3.42565273760521 E-92 & 3.42565273760521 E-92 & $3.42565273760521 \mathrm{E}-92$ & 3.42568583062114 E-92 & 3.42589465684648 E-92 \\
\hline 300 & 5.6824639994944 E-136 & 5.6824639994944 E-136 & 5.6824639994944 E-136 & 5.6824639994944 E-136 & $5.6825290903951 \mathrm{E}-136$ & $5.6827518702778 \mathrm{E}-136$ \\
\hline 350 & 8.0598618341338 E-158 & 8.0598618341338 E-158 & $8.0598618341338 \mathrm{E}-158$ & $8.0598618341338 \mathrm{E}-158$ & $8.0599564726193 \mathrm{E}-158$ & 8.0602190641050 E-158 \\
\hline 400 & 1.1910405364042 E-179 & 1.1910405364042 E-179 & $1.1910405364042 \mathrm{E}-179$ & $1.1910405364042 \mathrm{E}-179$ & $1.1910546339263 \mathrm{E}-179$ & 1.1910874374659 E-179 \\
\hline 500 & $2.8384990946186 \mathrm{E}-223$ & $2.8384990946186 \mathrm{E}-223$ & 2.8384990946186 E-223 & $2.8384990946186 \mathrm{E}-223$ & 2.8385323560887 E-223 & 2.8385904304095 E-223 \\
\hline 750 & 3.3717937435427 E-332 & $3.3717937435427 \mathrm{E}-332$ & 3.3717937435427 E-332 & 3.3717937435427 E-332 & 3.3718302948170 E-332 & $3.3718681256430 \mathrm{E}-332$ \\
\hline 900 & $1.6808087695655 \mathrm{E}-397$ & 1.6808087695655 E-397 & 1.6808087695655 E-397 & 1.6808087695655 E-397 & 1.6808260157552 E-397 & $1.6808399557005 \mathrm{E}-397$ \\
\hline 1000 & $5.0658373142902 \mathrm{E}-441$ & 5.0658373142902 E-441 & 5.0658373142902 E-441 & 5.0658373142902 E-441 & 5.0658874322192 E-441 & $5.0659222987817 \mathrm{E}-441$ \\
\hline 1500 & $1.6051526418476 \mathrm{E}-658$ & $1.6051526418476 \mathrm{E}-658$ & 1.6051526418476 E-658 & 1.6051526418476 E-658 & 1.6051660719573 E-658 & 1.6051708429826 E-658 \\
\hline 2000 & 6.4349079854541 E-876 & $6.4349079854541 \mathrm{E}-876$ & $6.4349079854541 \mathrm{E}-876$ & $6.4349079854541 \mathrm{E}-876$ & 6.4349547494925 E-876 & 6.4349630872006 E-876 \\
\hline 2500 & $2.934729424733 \mathrm{E}-1093$ & 2.934729424733 E-1093 & 2.934729424733 E-1093 & 2.934729424733 E-1093 & 2.934748347248 E-1093 & 2.934749611408 E-1093 \\
\hline 3000 & 1.452186663304 E-1310 & 1.452186663304 E-1310 & $1.452186663304 \mathrm{E}-1310$ & 1.452186663304 E-1310 & $1.452195105252 \mathrm{E}-1310$ & $1.452195010207 \mathrm{E}-1310$ \\
\hline 4000 & 4.147012701917 E-1745 & 4.147012701917 E-1745 & 4.147012701917 E-1745 & 4.147012701917 E-1745 & $4.147032983191 \mathrm{E}-1745$ & $4.147030640246 \mathrm{E}-1745$ \\
\hline 5000 & 1.347338831491 E-2179 & $1.347338831491 \mathrm{E}-2179$ & 1.347338831491 E-2179 & $1.347338831491 \mathrm{E}-2179$ & $1.347344554038 \mathrm{E}-2179$ & $1.347343503481 \mathrm{E}-2179$ \\
\hline 6000 & 4.749647324065 E-2614 & 4.749647324065 E-2614 & 4.749647324065 E-2614 & $4.749647324065 \mathrm{E}-2614$ & 4.749665229865 E-2614 & 4.749661067557 E-2614 \\
\hline 7000 & 1.771358837902 E-3048 & $1.771358837902 \mathrm{E}-3048$ & 1.771358837902 E-3048 & 1.771358837902 E-3048 & 1.771364860039 E-3048 & $1.771363235525 \mathrm{E}-3048$ \\
\hline 8000 & 6.884243980575 E-3483 & 6.884243980575 E-3483 & 6.884243980575 E-3483 & 6.884243980575 E-3483 & 6.884265343279 E-3483 & 6.884258946123 E-3483 \\
\hline 9000 & 2.761095088358 E-3917 & 2.761095088358 E-3917 & 2.761095088358 E-3917 & 2.761095088358 E-3917 & 2.761102983573 E-3917 & 2.761100426764 E-3917 \\
\hline 12000 & 2.031338494115 E-5220 & 2.031338494115 E-5220 & 2.031338494115 E-5220 & 2.031338494115 E-5220 & 2.031343229173 E-5220 & 2.031341443058 E-5220 \\
\hline
\end{tabular}

In present study, the relative error $\varepsilon \%$ of the activation energy have been computed by using Mathematica 8.0 software program. The obtained values of $\varepsilon \%$ are shown in Table 3 as a function of the parameter of $\mathrm{x}$. From the results in Table 3, it is seeming to be a marked effect of the parameter of $x$ on the sensitivity of the activation energy values.

\section{Numerical Results and Discussion}

In this study, we obtained general formulas for computing the Arrhenius functions by using arbitrary integral parameters. In this context, to test the derived equations for the Arrhenius integrals at the wide range of $x(\mathrm{E} / \mathrm{RT})$, the calculated results have been compared with the results of several methods proposed in the literature, and the results were listed in Tables $1-4$.

From the viewpoint of the computational efficiency and accuracy of our and various approximations, the Arrhenius functions were evaluated on the Mathematica 8.0 international mathematical software. The $\mu$ order exponential integral functions, appearing in equations 7,8 , and 9 , have been numerically evaluated. They appear tabulated in Table 1 for positive arguments up to 15 . Thus, the integral can be evaluated by simple computation for large arguments are common in many sterilization operations. As shown from Table 1, calculated results are well agreement with those of 
Deindoerfer and Humphrey [20]. The relative percent deviations of some Arrhenius integral approximations are shown in Table 2. As shown in Tables 2, the results are more accurate than other approximate formulas in the range of $1 \leq$ $x \leq 12000$. In Table 3 , it is shown the relative error (\%) from numerical results for some proposed approximations using Equations 13, 14, 15 and 16 as a function of the parameter $x(E / R T)$.

Values of the relative error (\%) for numerical results obtained by means of the different approximations methods as a function of the parameter $x(E / R T)$ and temperature $(T)$ using the equations $13,14,15$ and 16 are given in Table 4.

Table 3. The values of the relative error (\%) percentage deviations calculated by some proposed approximations using equations 13,14 , 15 and 16 at various $x$

\begin{tabular}{|c|c|c|c|c|}
\hline$x$ & $\begin{array}{l}\text { Coats and } \\
\text { Redfern [11] }\end{array}$ & $\begin{array}{c}\text { Doyle } \\
{[7]}\end{array}$ & $\begin{array}{c}\text { Horowitz and } \\
\text { Metzger [13] }\end{array}$ & $\begin{array}{c}\text { Van } \\
\text { Krevelen [6] }\end{array}$ \\
\hline 2 & -19.7176 & 71.4399 & 80.2823 & 30.2823 \\
\hline 5 & -4.7635 & 28.6034 & 35.2364 & 15.2364 \\
\hline 10 & -1.4698 & 12.7166 & 18.5302 & 8.5302 \\
\hline 20 & -0.4208 & 4.2046 & 9.5792 & 4.5792 \\
\hline 30 & -0.1971 & 1.2475 & 6.4696 & 3.1363 \\
\hline 50 & -0.0742 & -1.1715 & 3.9258 & 1.9258 \\
\hline 100 & -0.0192 & -3.0211 & 1.9808 & 0.9808 \\
\hline & $\infty$ & -4.9047 & $\sim 0$ & $\sim 0$ \\
\hline
\end{tabular}

Table 4. The values of the relative error $(\varepsilon \%)$ for the activation energy calculated by means of the equations $13,14,15$ and 16 as a function of the parameter $x(E / R T)$ and the temperature $(T)$

\begin{tabular}{|c|c|c|c|c|c|c|}
\hline$x \backslash \mathrm{T}$ & $\mathbf{3 0 0}$ & $\mathbf{4 0 0}$ & $\mathbf{6 0 0}$ & $\mathbf{8 0 0}$ & $\mathbf{1 0 0 0}$ & $\mathbf{1 2 0 0}$ \\
\hline 2 & 0.0019 & 0.0123 & 0.0227 & 0.0278 & 0.0309 & 0.0330 \\
\hline 5 & 0.0160 & 0.0201 & 0.0243 & 0.0263 & 0.0276 & 0.0284 \\
\hline 10 & 0.0202 & 0.0223 & 0.0244 & 0.0254 & 0.0260 & 0.0265 \\
\hline 20 & 0.0222 & 0.0233 & 0.0243 & 0.0248 & 0.0251 & 0.0253 \\
\hline 30 & 0.0229 & 0.0236 & 0.0242 & 0.0246 & 0.0248 & 0.0249 \\
\hline 50 & 0.0234 & 0.0237 & 0.0242 & 0.0244 & 0.0245 & 0.0246 \\
\hline 100 & 0.0237 & 0.0239 & 0.0241 & 0.0242 & 0.0243 & 0.0243 \\
\hline$\infty$ & 0.0241 & 0.0241 & 0.0241 & 0.0241 & 0.0241 & 0.0241 \\
\hline
\end{tabular}

\section{Conclusion}

It is known well that the Arrhenius integral does not have an exact analytical solution. In this context, in this study, a new analytical method for the Arrhenius integral based on incomplete gamma functions has been presented, which is simple, accurate and reliable. The validity of method proposed is tested by the results of other method in the literature, and the method show a good agreement. It can be concluded that the method may be used integral methods of thermal analysis.

\section{References}

[1] M.E. Brown, Introduction to Thermal Analysis: Techniques and Applications, second ed., Kluwer Academic Publishers, Boston, 2001.

[2] W.W. Wendlandt in Thermal Methods of Analysis, 2nd Edition, John Wiley \& Sons, New York, 1974.

[3] J. Cai, F. He, W. Yi and F. Yao, Chemical Engineering Journal 124 (2006) 15

[4] J. Cai, F. Yao, W. Yi and F. He, AIChE Journal 2006, 52 (4), 1554

[5] G.R. Heal, Thermochimica Acta 340-341 (1999) 69.

[6] D.W. Van Krevelen, C. Va Heerden and F.J. Huntjens, Fuel, 30 (1951) 253

[7] C.D. Doyle, Journal of Applied Polymer Science, 6 (1962) 639.

[8] C. D. Doyle, Anal Chem 1961, 33, 77.

[9] C. D. Doyle, J Appl Polym Sci 1961, 5, 285-292.

[10] C. D. Doyle, Nature 1965, 207, 290.

[11] A.W. Coats, J.P. Redfern, Nature 1964, 201, 68.

[12] A.W. Coats, J.P. Redfern, J Polym Sci, Part B: Polym Lett 1965, 3, 917.

[13] H.H. Horowitz, G. Metzger, Anal Chem 1963, 35, 1464-1468.

[14] J.R. MacCallum, J. Tanner, Eur Polym J 1970, 6, 1033-1039.

[15] J.R. MacCallum, J. Tanner, Eur Polym J 1970, 6, 907-917.

[16] G. Gyulai, E.J. Greenhow, Thermochimica Acta 1973, 6, 239-244.

[17] G. Gyulai, E.J. Greenhow, J Thermal Anal 1974, 6, 279-291.

[18] J.M.V. Capela, M.V. Capela, C.A. Ribeiro, J Math Chem 2009, 45, 769775

[19] I.S. Gradshteyn and I.M. Ryzhik (1980). Tables of Integrals, Series and Products. Academic Press, New York.

[20] F. H. Deindoerfer and A. E. Humphrey, Applied Microbiology 1959, 7(4), 256-264 